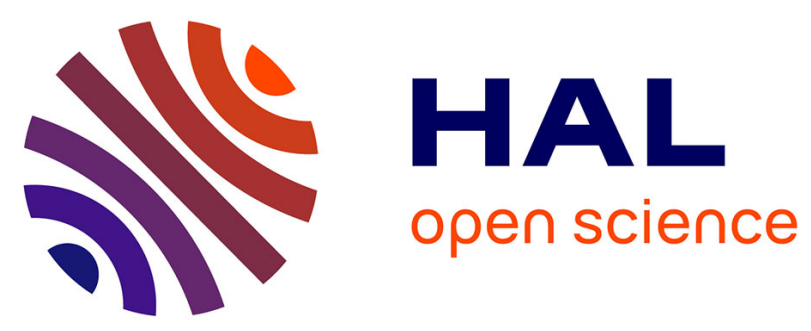

\title{
Caractéristiques électriques de couches minces isolantes d'oxydes et d'oxyfluorures déposés par pulvérisation cathodique
}

\author{
Guy Campet, Jean Claverie, Paul Hagenmuller, M. Perigord
}

\section{To cite this version:}

Guy Campet, Jean Claverie, Paul Hagenmuller, M. Perigord. Caractéristiques électriques de couches minces isolantes d'oxydes et d'oxyfluorures déposés par pulvérisation cathodique. Revue de Physique Appliquée, 1979, 14 (2), pp.415-420. 10.1051/rphysap:01979001402041500 . jpa-00244610

HAL Id: jpa-00244610 https://hal.science/jpa-00244610

Submitted on 1 Jan 1979

HAL is a multi-disciplinary open access archive for the deposit and dissemination of scientific research documents, whether they are published or not. The documents may come from teaching and research institutions in France or abroad, or from public or private research centers.
L'archive ouverte pluridisciplinaire $\mathbf{H A L}$, est destinée au dépôt et à la diffusion de documents scientifiques de niveau recherche, publiés ou non, émanant des établissements d'enseignement et de recherche français ou étrangers, des laboratoires publics ou privés. 


\title{
Caractéristiques électriques de couches minces isolantes d'oxydes et d'oxyfluorures déposés par pulvérisation cathodique
}

\author{
G. Campet, J. Claverie et P. Hagenmuller \\ Laboratoire de Chimie du Solide du C.N.R.S., Université de Bordeaux I,
} 351, cours de la Libération, 33405 Talence Cedex, France

M. Perigord,

La Radiotechnique, B.P. 301, 92156 Suresnes Cedex, France.

(Reçu le 10 août 1978, révisé le 23 octobre 1978, accepté le 27 octobre 1978)

\begin{abstract}
Résumé. - La conductivité électrique et les propriétés diélectriques de couches minces d'oxyde $\mathrm{Pb}_{2} \mathrm{Nb}_{2} \mathrm{O}_{7}$ et d'oxyfluorure $\mathrm{Pb}_{1,8} \mathrm{Zn}_{0,15} \mathrm{Na}_{0,05} \mathrm{Nb}_{2} \mathrm{O}_{6,95} \mathrm{~F}_{0,05}$ de structure pyrochlore ont été étudiées. La conductivité semble due à basse température $(T<300 \mathrm{~K})$ à un phénomène de hopping entre impuretés de type $\mathrm{p}$ ou $\mathrm{n}$, à haute température $(T>300 \mathrm{~K})$ à une conduction ionique. Les constantes diélectriques des couches minces $\left(\varepsilon_{\mathrm{r}}^{\prime} \simeq 30\right)$ sont inférieures à celles des céramiques correspondantes $\left(\varepsilon_{\mathrm{r}}^{\prime} \simeq 200\right)$, résultat qui s'explique par le caractère amorphe des films obtenus. Les couches minces de $\mathrm{Pb}_{2} \mathrm{Nb}_{2} \mathrm{O}_{7}$, qui comportent de faibles pertes diélectriques $\left(\operatorname{tg} \delta \simeq 30 \times 10^{-4}\right.$ ) sont susceptibles d'utilisation dans des dispositifs microélectroniques.
\end{abstract}

Abstract. - The electric conductivity and dielectric properties of thin films of the pyrochlores $\mathrm{Pb}_{2} \mathrm{Nb}_{2} \mathrm{O}_{7}$ and $\mathrm{Pb}_{1.8} \mathrm{Zn}_{0.15} \mathrm{Na}_{0.05} \mathrm{Nb}_{2} \mathrm{O}_{6.95} \mathrm{~F}_{0.05}$ have been investigated. The conductivity is due at low temperature $(T<300 \mathrm{~K})$ to a hopping mechanism between impurities of $p$ and $n$ type, and at high temperature $(T>300 \mathrm{~K})$ to ionic conductivity. The permittivities of the thin films $\left(\varepsilon_{\mathrm{r}}^{\prime} \simeq 30\right)$ are smaller than those of corresponding ceramics $\left(\varepsilon_{\mathrm{r}}^{\prime} \simeq 200\right)$, which may be accounted for by the amorphous character of the layers. $\mathrm{Pb}_{2} \mathrm{Nb}_{2} \mathrm{O}_{7}$ films, which have weak dielectric losses $\left(\operatorname{tg} \delta \simeq 30 \times 10^{-4}\right)$, are able to be used in microelectronic devices.

La miniaturisation des dispositifs électroniques est à l'origine des études entreprises pour découvrir des matériaux nouveaux de haute permittivité susceptibles d'être utilisés en couches minces, sans pertes appréciables.

Pour pallier les inconvénients des matériaux existants, on utilise généralement soit des mélanges constitués d'un oxyde ferroélectrique et d'un matériau paraélectrique soit des phases pures dérivant de l'oxyde ferroélectrique par diverses substitutions cationiques $[1,2]$. On obtient dans les deux cas des céramiques de propriétés diélectriques convenables.

Nous avons récemment montré que ces performances peuvent être améliorées de façon sensible. En effet les matériaux diélectriques que nous avons obtenus par substitution de l'oxygène par le fluor dans des oxydes ferroélectriques à bas point de Curie présentaient des caractéristiques diélectriques remarquables et étaient également susceptibles d'être déposés en couches minces par pulvérisation cathodique $[3,4,5,6]$.
L'étude des caractéristiques électriques de ces couches minces devrait permettre de mieux comprendre les corrélations entre les conditions de préparation et les propriétés diélectriques de l'isolant, conduisant ainsi à une fiabilité des caractéristiques électriques compatible avec une utilisation en microélectronique.

1. Choix des matériaux. - Notre choix s'est porté sur l'oxyde $\mathrm{Pb}_{2} \mathrm{Nb}_{2} \mathrm{O}_{7}$ de structure pyrochlore rhomboédrique dont la température de Curie $(15 \mathrm{~K})$ est particulièrement basse [7].

L'abaissement de charge anionique résultant de la substitution de l'oxygène par le fluor est compensé par le remplacement d'une quantité équivalente de plomb par le sodium, ce qui conduit à la solution solide : $\mathrm{Pb}_{2-x} \mathrm{Na}_{x} \mathrm{Nb}_{2} \mathrm{O}_{7-x} \mathrm{~F}_{x}$. L'étude diélectrique montre que la composition optimale qui allie une permittivité élevée à de faibles pertes $\left(\varepsilon_{\mathrm{r}}^{\prime}=160\right.$; $\operatorname{tg} \delta=10^{-3}$ ) correspond à $x=0,05$ [3]. Ces performances sont encore améliorées si on remplace une 
autre partie des ions $\mathrm{Pb}^{2+}$ par des ions $\mathrm{Zn}^{2+}$ [8]. Il s'avère que l'oxyfluorure

$\mathrm{Pb}_{1,8} \mathrm{Zn}_{0,15} \mathrm{Na}_{0,05} \mathrm{Nb}_{2} \mathrm{O}_{6,95} \mathrm{~F}_{0,05}$

$\left(\varepsilon_{\mathrm{r}}^{\prime}=200 ; \operatorname{tg} \delta<3 \times 10^{-4}\right)$ est le diélectrique de classe I qui possède les meilleures performances actuellement connues (classe I implique de faibles pertes et un faible coefficient de température).

2. Techniques expérimentales. - ELABORATION DES MATÉRIAUX. - L'oxyde et l'oxyfluorure s'obtiennent par synthèse directe à partir d'un mélange d'oxydes et de fluorure selon le schéma réactionnel suivant :

$$
\begin{aligned}
& (2-x-y) \mathrm{PbO}+x \mathrm{NaF}+y \mathrm{ZnO}+ \\
& \quad+\mathrm{Nb}_{2} \mathrm{O}_{5} \rightarrow \mathrm{Pb}_{2-x-y} \mathrm{Na}_{x} \mathrm{Zn}_{y} \mathrm{Nb}_{2} \mathrm{O}_{7-x} \mathrm{~F}_{x} .
\end{aligned}
$$

Pour $x=y=0$ on obtient $\mathrm{Pb}_{2} \mathrm{Nb}_{2} \mathrm{O}_{7}$, pour $x=0,05$ et $y=0,15$ l'oxyfluorure

$\mathrm{Pb}_{1,8} \mathrm{Zn}_{0,15} \mathrm{Na}_{0,05} \mathrm{Nb}_{2} \mathrm{O}_{6,95} \mathrm{~F}_{0,05}$.

Les mélanges stœechiométriques sont chauffés à $900{ }^{\circ} \mathrm{C}$ sous argon, pendant 8 heures, en tubes d'or scellés, puis trempés. Les produits se présentent sous forme de poudres bien cristallisées de couleur blancjaunâtre.

Ces poudres sont alors compactées sous une pression de $5 \mathrm{kbar}$ afin d'obtenir des pastilles cylindriques de $13 \mathrm{~mm}$ de diamètre et de $2 \mathrm{~mm}$ d'épaisseur, puis frittées sous argon pendant 15 heures, à $950^{\circ} \mathrm{C}$ en tube d'or scellé. Les pastilles obtenues sont dures, non poreuses et de compacité supérieure à $85 \%$.

2.2 Dépot des matériauX en COUCHeS MinCes. Les couches minces de diélectrique de $4000 \AA$ d'épaisseur environ, sont obtenues par pulvérisation cathodique radio-fréquence, la pastille précédente jouant le rôle de cible.

Le dépôt effectué à la vitesse de $150 \AA / \mathrm{min}$. est obtenu pour une puissance de 500 watts. L'atmosphère de l'enceinte, dont la pression est de $10^{-3}$ torr, est constituée d'un mélange de $90 \%$ d'argon et de $10 \%$ d'oxygène en volumes; la présence de ce dernier gaz est destinée à éviter la formation de couches déficitaires en oxygène $[6,9]$.

L'épaisseur des dépôts est déterminée par la méthode du microscope interférentiel en lumière polarisée [10].

L'analyse radiocristallographique montre que les couches sont amorphes.

Leur composition déterminée par analyse nucléaire montre que les couches $\mathrm{C}_{1}$ obtenues à partir de l'oxyde $\mathrm{Pb}_{2} \mathrm{Nb}_{2} \mathrm{O}_{7}$ présentent sensiblement la même composition que le produit de départ. En revanche les couches $\mathrm{C}_{2}$ provenant de la pulvérisation de l'oxyfluorure $\mathrm{Pb}_{1,8} \mathrm{Zn}_{0,15} \mathrm{Na}_{0,05} \mathrm{Nb}_{2} \mathrm{O}_{6,95} \mathrm{~F}_{0,05}$ sont très déficitaires en plomb, le bombardement ionique ayant éjecté les éléments légers $(\mathrm{Na}$ et $\mathrm{F}$ ) préférentiellement à l'élément lourd $(\mathrm{Pb})$. Les couches $\mathrm{C}_{2}$ ont approximativement la composition :

$\mathrm{PbNa}_{0,20} \mathrm{Zn}_{0,15} \square_{0,65} \mathrm{Nb}_{2} \mathrm{O}_{6,15} \mathrm{~F}_{0,2} \square_{0,65}$

elles sont donc fortement lacunaires par rapport aux pyrochlores-types $\mathrm{A}_{2} \mathrm{~B}_{2} \mathrm{X}_{7}$.

2.3 Mesure des CARACTÉRISTIQUES ÉLECTRIQUES. Les cellules sont constituées d'un substrat isolant sur lequel sont déposées successivement une électrode métallique, la couche mince de diélectrique et une contre-électrode. Des fils de cuivre soudés sur les électrodes à l'aide d'une laque d'argent Degussa permettent le raccordement entre la cellule et les appareils de mesure.

Les substrats sont des lames de verre, dégraissées à l'acétone, décapées à l'eau régale, rincées et séchées.

Les électrodes sont obtenues par évaporation sous vide d'un fil d'aluminium, choisi en raison de sa faible résistivité et de sa bonne adhésion au verre. L'épaisseur des électrodes est de $150 \AA$, celle du diélectrique de $4000 \AA$.

Compte tenu des positions respectives des électrodes, la surface du diélectrique qui leur fait face est de $4 \times 7 \mathrm{~mm}^{2}$.

Le comportement électrique des cellules en fonction de la température est étudié sous tensions continue et alternative. Nous avons fixé les cellules sur un bloc de cuivre à l'intérieur duquel est enroulé un themocoax permettant d'obtenir une température uniforme.

Les tensions continues appliquées aux cellules sont délivrées par une alimentation stabilisée, l'intensité du courant qui les traverse est mesurée à l'aide d'un picoampèremètre Keitley 416.

Les mesures de capacité et de facteurs de pertes sous tension alternative sont réalisées par la méthode du pont de Schering à l'aide d'un pont de capacité General Radio 716.

3. Résultats expérimentaux et discussion. -3.1 ETUDE DES MODES DE CONDUCTION SOUS TENSION CONTINUE. - Les courbes de variation thermique du logarithme de la densité de courant $J_{c}$ qui traverse les cellules $C_{1}$ et $C_{2}$ laissent apparaître deux phénomènes distincts de part et d'autre de $T=340 \mathrm{~K}$, température pour laquelle se produit un changement de pente (Figs. 1 et 2).

Les très faibles énergies d'activation observées à basse température résultent probablement d'une conduction par hopping électronique due à la présence d'impuretés de type $n$ et $p$, phénomène caractéristique des matériaux amorphes ou mal cristallisés.

Lorsque la température est supérieure à $340 \mathrm{~K}$, les points expérimentaux se placent sur une droite, qui correspond à l'équation classique d'Arrhenius : $J=J_{0} \mathrm{e}^{-E / k T}$. L'énergie d'activation propre au phénomène est égale à $E_{1}=1,02 \pm 0,02 \mathrm{eV}$ pour $\mathrm{C}_{1}$ et à $E_{2}=0,86 \pm 0,02 \mathrm{eV}$ pour $\mathrm{C}_{2}$. On peut penser 


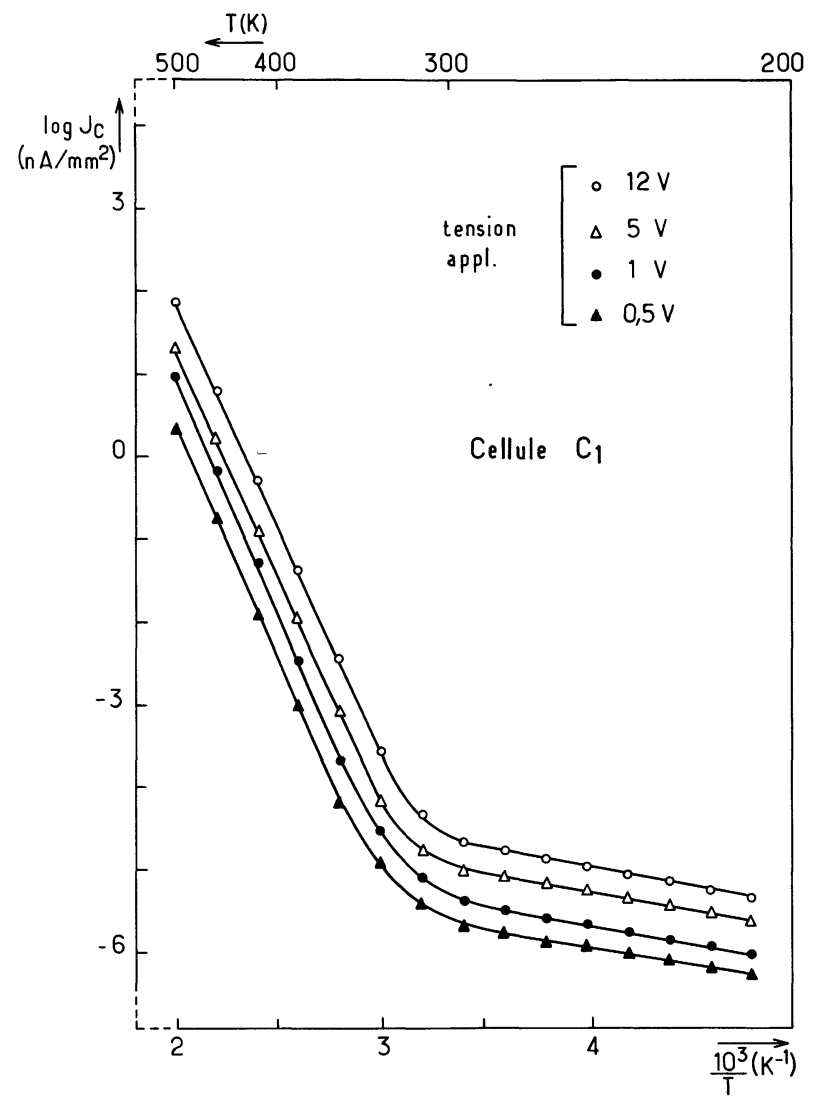

Fig. 1. - Variation en fonction de $10^{3} / T$ du logarithme de la densité de courant parcourant la cellule $\mathrm{C}_{1}$.

[Variation of $\log J_{\mathrm{c}} v s 10^{3} / T$ in the cell $\mathrm{C}_{1}$.]

que dans cette zone de température se produit un phénomène de conduction ionique lié à la présence de lacunes au sein des films déposés.

Les figures 3 et 4 montrent que la densité de courant proportionnelle à la tension lorsque celle-ci reste inférieure à $0,6 \mathrm{~V}$ environ (Fig. 3) varie exponentiellement avec elle à tension plus élevée (Fig. 4). Une conductivité électronique par effet Schootky ou Poole-Frenkel est donc à exclure. Elle impliquerait en effet que le logarithme de l'intensité soit proportionnel à la racine carrée de la tension appliquée, ce qui n'est pas le cas.

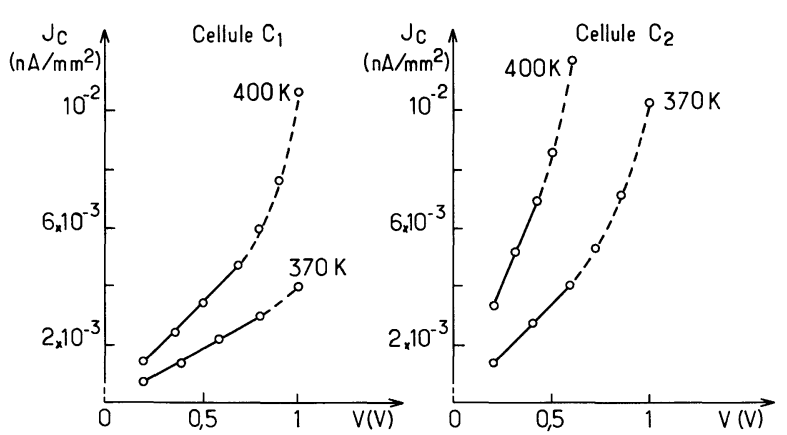

Fig. 3. - Variation de la densité du courant parcourant les cellules en fonction de la tension appliquée.

[Variation of the current $v s$ applied potential for the cells $\mathrm{C}_{1}$ and $\mathrm{C}_{2}$.]

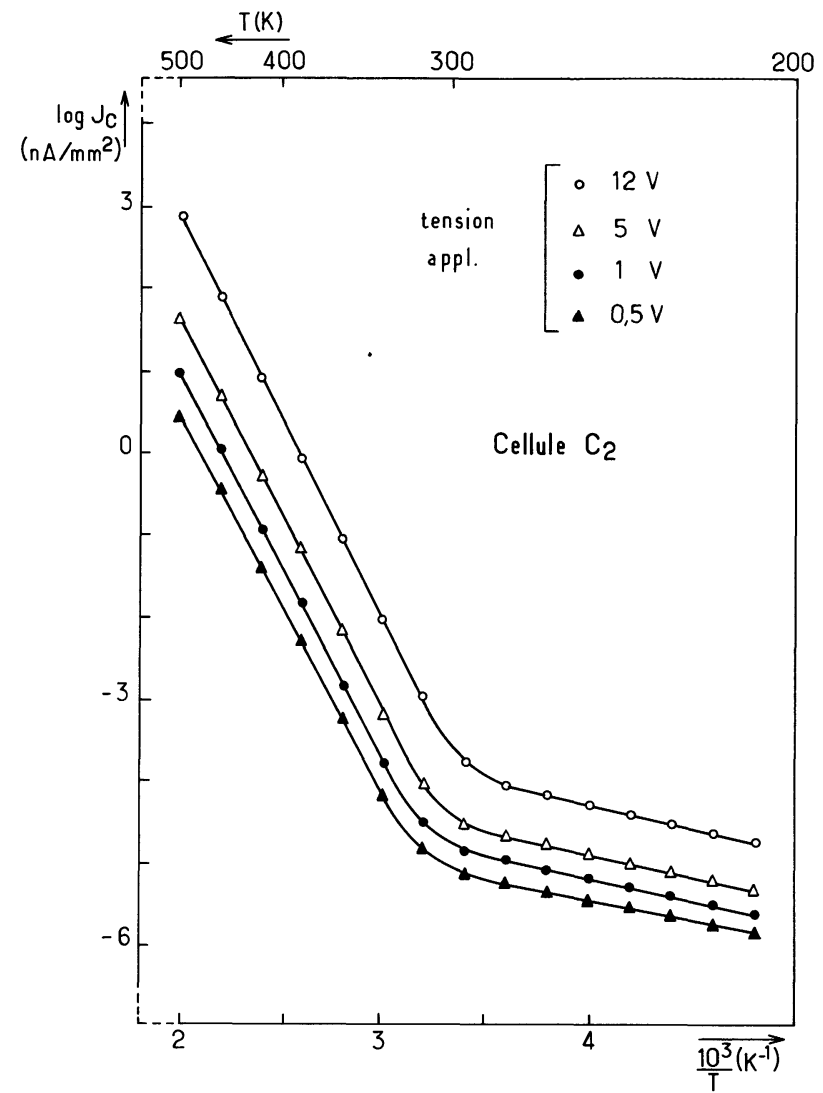

Fig. 2. - Variation en fonction de $10^{3} / T$ du logarithme de la densité du courant parcourant la cellule $\mathrm{C}_{2}$.

[Variation of $\log J_{\mathrm{c}} v s 10^{3} / T$ in the cell $\mathrm{C}_{2}$.]

La densité de courant est plus forte et l'énergie d'activation plus faible pour les couches minces $\mathrm{C}_{2}$ que pour les films $\mathrm{C}_{1}$. Un tel comportement ne saurait surprendre. $\mathrm{La}$ composition du diélectrique $\mathrm{C}_{2}$ entraîne l'existence d'un plus grand nombre de porteurs, donc une conductivité accrue liée à la grande mobilité d'ions tels que $\mathrm{Na}^{+}$ou $\mathrm{F}^{-}$présents dans la couche $\mathrm{C}_{2}$, mobilité favorisée par le caractère fortement lacunaire du réseau.

La tension maximale $V_{\mathrm{c}}$ que l'on peut appliquer aux couches minces $\mathrm{C}_{1}$ et $\mathrm{C}_{2}$ d'épaisseur $e=4 \times 10^{-5} \mathrm{~cm}$ sans les détériorer est de 30 volts environ à tempé-

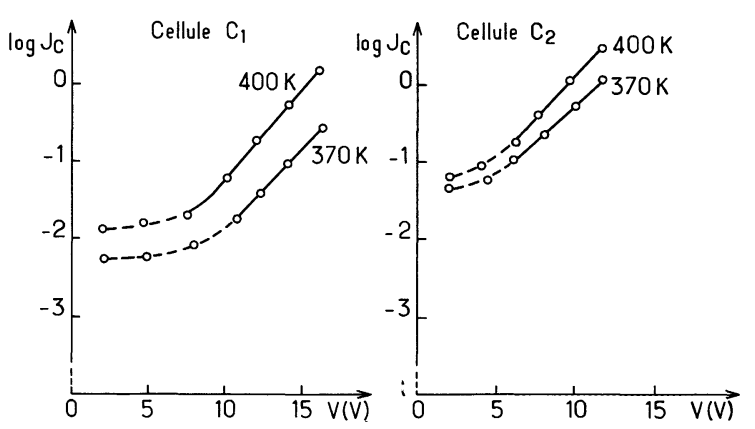

Fig. 4. - Variation du logarithme de la densité du courant parcourant les cellules en fonction de la tension appliquée.

[Variation of $\log J_{\mathrm{c}} v s$ applied potential for the cells $\mathrm{C}_{1}$ and $\mathrm{C}_{2}$.] 
rature ambiante. Le champ de claquage peut alors être évalué à $7,5 \times 10^{5}$ volts $\mathrm{cm}^{-1}$. C'est une valeur particulièrement élevée.

\subsection{ETUDE DES MODES DE CONDUCTION SOUS TENSION} ALTERNATIVE. - La variation en fonction de la fréquence de la permittivité et du logarithme du facteur de pertes des cellules est représentée aux figures 5 et 6 .

Ces deux grandeurs demeurent pratiquement constantes si la fréquence est comprise entre $5 \times 10^{2} \mathrm{~Hz}$ et $5 \times 10^{3} \mathrm{~Hz}$. Quand celle-ci est inférieure à $5 \times 10^{2} \mathrm{~Hz}$ et décroît, la permittivité et le facteur de pertes augmentent d'autant plus rapidement que la température est plus élevée; ce phénomène est plus sensible pour $C_{2}$ que pour $C_{1}$.

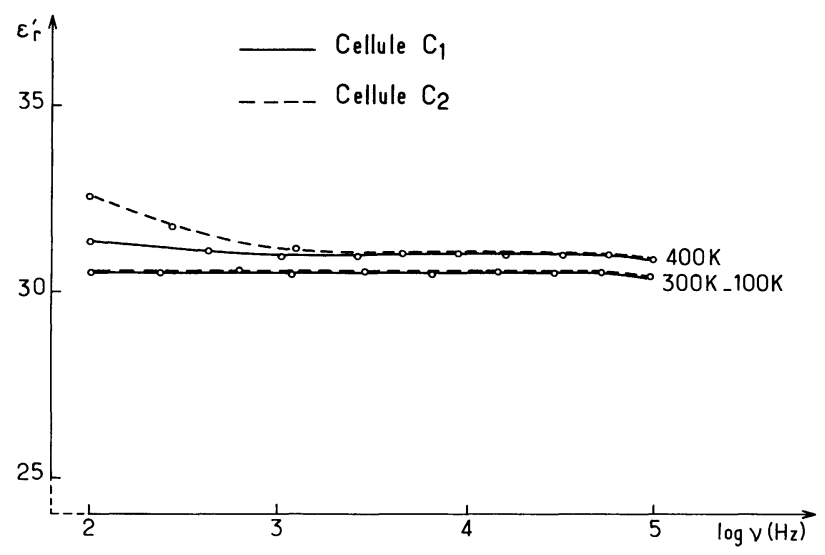

Fig. 5. - Variation de la permittivité des cellules en fonction du logarithme de la fréquence.

[Permittivity variation of the cells as a function of $\log v$.]

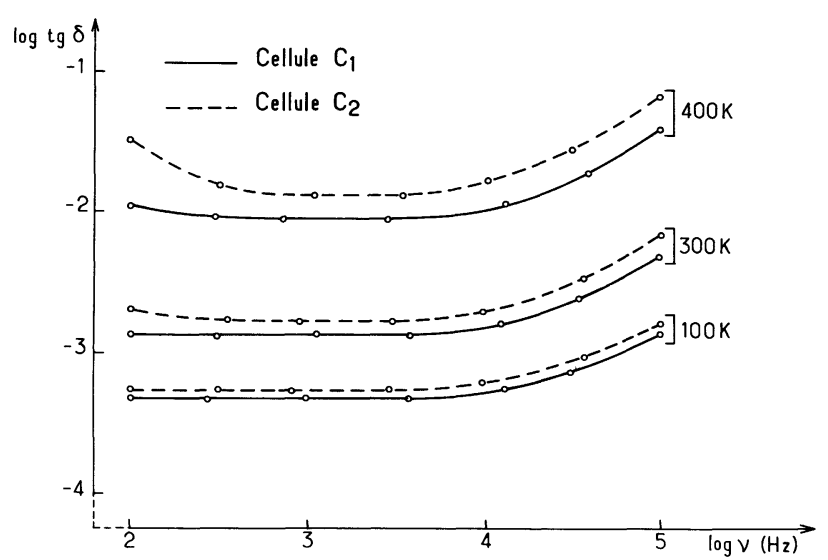

Fig. 6. - Variation du logarithme du facteur de pertes en fonction du logarithme de la fréquence.

[Frequency dependence of dielectric losses for $C_{1}$ and $C_{2}$ cells.]

A température ordinaire la permittivité des isolants $\mathrm{C}_{1}$ et $\mathrm{C}_{2}$ peut être évaluée à $\varepsilon_{\mathrm{r}}^{\prime} \simeq 30 \pm 3$ à partir des données précédentes. Cette valeur est très inférieure à celle que présente à l'état massif l'oxyde $\mathrm{Pb}_{2} \mathrm{Nb}_{2} \mathrm{O}_{7}$ pour lequel $\varepsilon_{\mathrm{r}}^{\prime} \simeq 130$. D'autre part les céramiques de même composition que les couches minces $C_{2}$ pré- sentent une permittivité de l'ordre de 100 . On peut penser que le relâchement structural inhérent au passage de l'état cristallisé à l'état amorphe est, en partie tout au moins, responsable de l'abaissement de la permittivité.

Nous avons proposé antérieurement pour expliquer les valeurs élevées de la permittivité des niobates de plomb, un mécanisme de polarisabilité ionique dû à la déformation élastique de l'ion $\mathrm{Pb}^{2+}$ sous l'action d'un champ électrique [3]. L'ion $\mathrm{Pb}^{2+}$ comporte en effet un doublet électronique non engagé (lone pair) facilement déformable qui lui confère un moment dipolaire spontané; les anions situés au voisinage de ces dipôles subissent une interaction électrostatique : ces déplacements anioniques privilégiés favorisent à leur tour l'apparition de dipôles ioniques. L'alignement de ces dipôles se fait suivant un mécanisme faisant intervenir la covalence des liaisons métal-oxygène [13]. On comprend dès lors que l'absence d'ordre à longue distance dans un matériau amorphe perturbera à la fois l'apparition des dipôles ioniques et leur alignement. C'est le cas de nos couches minces, dont se trouve ainsi expliqué l'abaissement de permittivité.

Pour les fréquences inférieures à $5 \times 10^{2} \mathrm{~Hz}$ et pour des températures élevées, la légère augmentation de la permittivité et du facteur de pertes observée pour les films $\mathrm{C}_{2}$ notamment conduit à penser qu'il se produit, à basse fréquence un mécanisme de relaxation [12]. Il peut être attribué à la formation de charges d'espace, d'origine ionique, dues à l'accumulation d'ions tels que $\mathrm{F}^{-}$ou $\mathrm{Na}^{+}$soit autour d'un défaut (lacune, impureté...) soit à l'interface électrodediélectrique. Ces résultats sont à rapprocher de ceux établis précédemment en courant continu.

Par ailleurs l'accroissement des pertes observé pour $v>5 \times 10^{3} \mathrm{~Hz}$, qui est accompagné d'une légère diminution de la capacité, n'est probablement pas une propriété intrinsèque du diélectrique. On a montré en effet que le comportement de la capacité et du facteur de pertes des cellules, pour des fréquences élevées, est fonction d'une part de la résistance des électrodes, d'autre part de la nature et de la localisation sur les électrodes des contacts reliant les cellules aux appareils de mesure [11].

La figure 7 représente les variation du logarithme du courant de pertes diélectriques $J_{\mathrm{f}}$ en fonction de l'inverse de la température absolue, pour une fréquence de $1 \mathrm{kHz}$ et une tension de $300 \mathrm{mV}$.

A température suffisamment élevée $(T>330 \mathrm{~K}$ environ), les points expérimentaux s'alignent : $J_{\mathrm{f}}$ peut alors s'écrire sous la forme $J_{\mathrm{f}}=J_{0} \mathrm{e}^{-E / k T}$. L'énergie d'activation est égale à $0,52 \pm 0,02 \mathrm{eV}$ pour $C_{1}$ et à $0,48 \pm 0,02 \mathrm{eV}$ pour $\mathrm{C}_{2}$.

Pour des températures inférieures à $280 \mathrm{~K}$ nous observons également des droites, mais leur pente est très faible; les énergies d'activation correspondantes sont inférieures à $0,04 \mathrm{eV}$.

Le graphe 7 présente une grande similitude avec les 


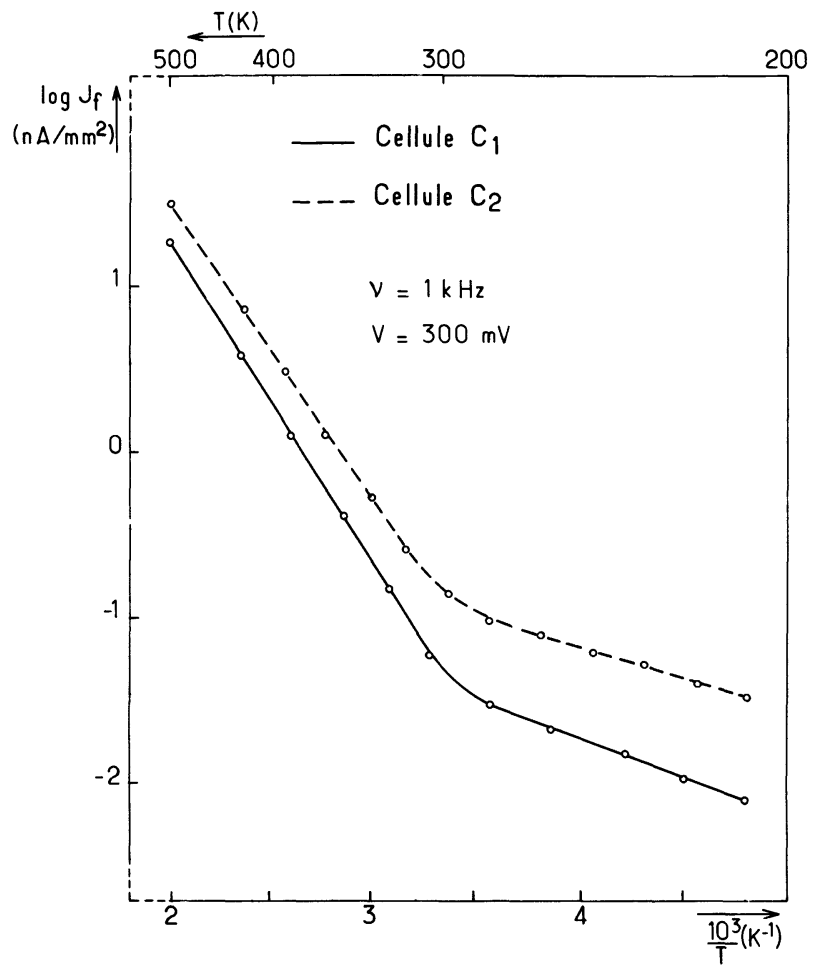

Fig. 7. - Variation du logarithme de la densité de courant de pertes diélectriques dans les cellules $C_{1}$ et $C_{2}$ en fonction de $10^{3} / T$.

[Temperature dependence of the loss current for $C_{1}$ and $C_{2}$ cells.]

graphes 1 et 2. Nous utiliserons donc le même raisonnement que pour une tension continue en supposant que la conduction est ionique dans le domaine de haute température $(T>330 \mathrm{~K})$ et du type hopping électronique dans le domaine de basse température $(T<.280 \mathrm{~K})$. La densité de courant mesurée en courant alternatif est nettement supérieure toutefois à celle mesurée en courant continu $\left(J_{\mathrm{f}}>J_{\mathrm{c}}\right)$. Ceci est dû au fait que les deux phénomènes n'ont pas tout à fait la même origine :

Sous une tension continue le courant est dû uniquement à la translation des porteurs à travers la masse du diélectrique; les dipôles existant au sein du diélectrique ou qui sont induits par le champ s'orientent, mais influent peu sur la conductivité.

Sous tension alternative la rotation des dipôles ou les déplacements de charge entre sites voisins qui se produisent, engendrent des pertes supplémentaires qui se traduisent par un accroissement de la valeur de $J_{\mathrm{f}}$.

4. Conclusions. - Les mesures électriques effectuées sur des structures électrode ( $\mathrm{Al}$ )-diélectrique-électrode (Al) ont permis de discerner les principaux mécanismes de conduction au sein de couches minces du pyrochlore $\mathrm{Pb}_{2} \mathrm{Nb}_{2} \mathrm{O}_{7}$ et d'un dérivé de substitution oxyfluoré. A température suffisamment basse les couches minces semblent être le siège d'un phénomène de hopping dû à la présence simultanée d'impuretés de type $\mathrm{n}$ et $\mathrm{p}$. A température plus élevée une conduction ionique apparaît, elle est particulièrement marquée dans les films $\mathrm{C}_{2}$; elle proviendrait essentiellement du déplacement des ions de petite taille faiblement chargés.

Les permittivités relatives des films déposés sont de l'ordre de $30 \pm 3$. Celles des céramiques de même composition étaient au moins trois fois plus élevées; cet écart semble résulter de l'absence d'ordre à longue distance inhérent au passage de l'état cristallisé à l'état amorphe.

Dans la perspective d'une utilisation en microélectronique, on peut toutefois remarquer que les films $\mathrm{C}_{1}$ à base de $\mathrm{Pb}_{2} \mathrm{Nb}_{2} \mathrm{O}_{7}$ possèdent une permittivité et un facteur de pertes très stables en température et en fréquence qui conduisent à penser qu'ils sont susceptibles d'améliorer la qualité des condensateurs en couches minces actuellement utilisés.

La comparaison de leurs propriétés avec celles que comportent les dépôts de monoxyde de silicium revêt un grand intérêt : les condensateurs en couches minces actuellement utilisés dans les circuits hybrides sont en effet essentiellement à base de "SiO ». Le tableau I rassemble les principales propriétés de ces matériaux.

Tableau I
Caractéristiques

Constante diélectrique

Tension de service pour $e=4000 \AA$

Coefficient de température de 20 à $70^{\circ} \mathrm{C}$

Tangente de l'angle de pertes pour $v=1 \mathrm{kHz}$ Résistivité à $20^{\circ} \mathrm{C}$

$\begin{array}{cc}\text { Nature du dépôt } \\ \mathrm{SiO} & \mathrm{Pb}_{2} \mathrm{Nb}_{2} \mathrm{O}_{7} \\ \frac{7}{7} & -\overline{30}\end{array}$

$40 \mathrm{~V}$ $30 \mathrm{~V}$
Remerciements. - Les auteurs expriment leurs sincères remerciements à M. Pompei (R.T.C. Compelec à Suresnes) dans le service duquel ont été réalisés les dépôts en couches minces, à MM. Barrière, Danto et Salardenne (Laboratoire d'Electronique et de Physique du Solide de l'Université de Bordeaux I) pour leur efficace collaboration, et à la D.G.R.S.T. pour l'aide matérielle apportée à ce travail (contrat C.C.M. 74.7.0425).

\section{Bibliographie}

[1] Anderson, J. C., Diélectriques (Dunod Paris), 1966, p. 187.

[2] Jouenne, C. A., Céramique générale (Gauthier-Villars, Paris) 1966, p. 159.
[3] Campet, G., Claverie, J., Perigord, M., Ravez, J., Portier, J. et Hagenmuller, P., Mat. Res. Bull. 9 (1974) 1589.

[4] Brevet ANVAR no 73.373 (1973). 
[5] Claverie, J., Campet, G., Ravez, J., Perigord, M., Portier, J. et Hagenmuller, P., C.R. Hebd. Séan. Acad. Sci. 280C (1975) 801.

[6] Pompei, J., Campet, G., Claverie, J. et Hagenmuller, P., Bull. Soc. Franc. Cér. 117 (1977) 15.

[7] Smolensky, C. A. et Ogranowskaya, A. J., Sov. Phys. Solid State 1 (1959) 1429.

[8] CAMPet, G., Thèse doctorat ès sciences physiques, Université de Bordeaux-I (1975).
[9] POMPEI, J., L'onde électrique 44 (1964) 1287.

[10] Brossel, J., J. Proc. Phys. Soc. 59 (1947) 224.

[11] Perigord, M., Thèse doctorat ès sciences physiques, Université de Bordeaux-I (1975).

[12] Harrop, P. J. et Campbell, D. S., Thin Solid Films 2 (1968) 273.

[13] Chaminade, J. P., Thèse doctorat ès sciences physiques, Université de Bordeaux-I (1974). 